\title{
Interhemispheric Paracallosal Approach for an Arteriovenous Malformation of the Atrium: A Novel Technique
}

\author{
Edison P. Valle-Giler, MD, ${ }^{1,2}$ Edna E. Gouveia, MD, ${ }^{1}$ Roger D. Smith, MD $^{1,2}$ \\ ${ }^{1}$ Department of Neurosurgery, Ochsner Clinic Foundation, New Orleans, LA ${ }^{2}$ The University of Queensland Faculty of Medicine, Ochsner \\ Clinical School, New Orleans, LA
}

Background: Intraventricular lesions present a surgical challenge because of the complexity of the ventricular anatomy, various perforating arteries, and eloquent brain areas surrounding the system. The ventricular atrium is particularly challenging because of the cingulate gyrus, corpus callosum, optic pathways, and significant vascular structures. We present the case of a patient for whom we used a new surgical approach to reach a lesion in the ventricular atrium.

Case Report: A 26-year-old male presented with an intraventricular hemorrhage, acute hydrocephalus, and a grade III arteriovenous malformation (AVM) in the atrium of the left lateral ventricle. We approached the AVM through a posterior parietooccipital paracallosal interhemispheric approach. Instead of transecting the cingulate cortex as is traditionally done, we gently retracted the cingulate gyrus and made a small paracallosal incision to reach the atrium of the left lateral ventricle. The surgery was uneventful. The patient recovered well and was discharged home on postoperative day 3 without any deficits.

Conclusion: This case illustrates a novel variation to an established approach to the ventricular atrium. With this technique, the surgeon minimizes disruption of brain tissue and thereby avoids the associated postoperative deficits associated with traditional approaches (transcortical, transcingulate, and conventional transcallosal). Tractography studies and a large cohort of patients are necessary to ensure the reproduction of good outcomes.

Keywords: Arteriovenous malformations, central nervous system vascular malformations, corpus callosum, gyrus cinguli, microsurgery, surgical procedures-operative

Address correspondence to Edison P. Valle-Giler, MD, Department of Neurosurgery, Ochsner Clinic Foundation, 1514 Jefferson Hwy., New Orleans, LA 70121. Tel: (504) 842-4033. Email: edison.vallegiler@ochsner.org

\section{INTRODUCTION}

An arteriovenous malformation (AVM) is a type of vascular anomaly in which, among a tangle of vessels, blood flows directly from arteries to draining veins without an intervening capillary network. This direct arteriovenous connection causes a high-flow environment that makes AVMs quite fragile and prone to bleeding. The risk of bleeding is lifelong, and the risk of hemorrhage has been calculated to be $2 \%-4 \%$ per year. $^{1}$

Treatment modalities for AVMs include surgery, stereotactic radiosurgery, and embolization. Microsurgical resection is still the treatment of choice, as it is immediately curative in approximately $50 \%$ of patients with an AVM. ${ }^{2}$ However, surgery is only offered if the location and type of AVM are amenable to surgical intervention.

The ventricular system of the brain is a difficult location for surgery and an area in which AVMs can be present, although rarely. Surgical approaches to these intraventricular AVMs must acknowledge the numerous perforating arteries supplying critical subcortical regions and the multidirec- tional white matter tracts associated with the ventricular system. The atrium of the lateral ventricle is a particularly difficult location to access in surgical interventions. Numerous microsurgical approaches have been described for accessing the atrium, including transcortical at the superior parietooccipital junction, posterior transcallosal, parietooccipital interhemispheric transcingulate, and lateral transtemporal at the temporoparietal junction or at the middle/inferior temporal gyrus, each with its own risks and complications. ${ }^{3-5}$

We present a new surgical variation of the interhemispheric approach to the atrium of the lateral ventricle. This approach avoids the traditional transcingulate, transcortical, and transcallosal routes and thus also avoids the possible deficits associated with the disruption of these structures.

\section{CASE REPORT}

A 26-year-old male presented to an outside hospital with severe, sudden-onset headache and loss of consciousness; computed tomography (CT) scan of the brain showed intraventricular hemorrhage with obstructive hydrocephalus 

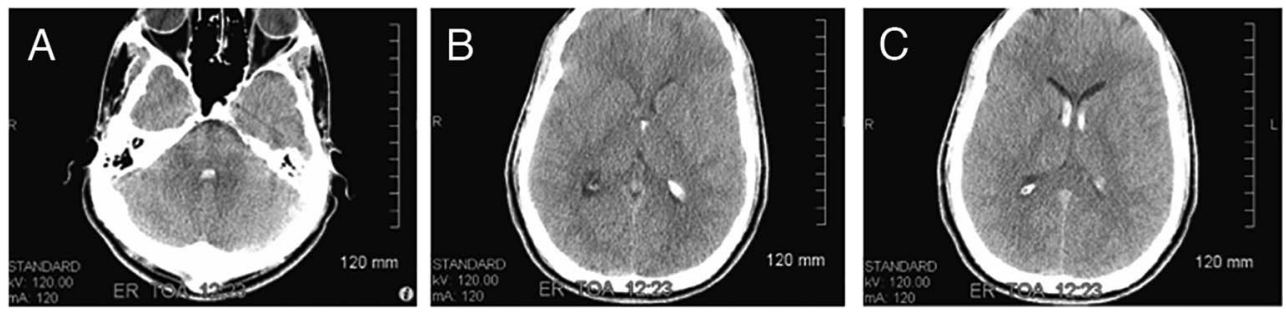

Figure 1. Computed tomography (CT) on hospital day 1 demonstrates (A and $B$ ) intraventricular hemorrhage, and (C) a follow-up CT 24 hours after onset of symptoms shows hydrocephalus.

(Figure 1). The patient was emergently transferred to our hospital. On initial examination at arrival to the hospital, the patient was awake without any focal neurologic deficits. An external ventricular drain (EVD) was placed emergently as the patient subsequently became obtunded. Further workup with magnetic resonance angiography showed a SpetzlerMartin grade III AVM in the left lateral ventricle (Figure 2). Digital subtraction angiography confirmed the $3 \mathrm{~mm} \times 2 \mathrm{~mm}$ left atrial AVM (Figure 3). The patient was kept in the intensive care unit to clear the intraventricular hemorrhage and stabilize clinically for 7 days.

On hospital day 8, the patient was taken to the operating room. Femoral arterial access was obtained prior to positioning for intraoperative angiography. The patient was placed in the prone position with the head extended 20 degrees, and the neuronavigation system was synced with the patient's computed tomography angiography. An occipital-based horseshoe incision was made perpendicular to the location of the AVM using neuronavigation as a reference. A craniotomy centered over the parietooccipital sagittal sinus was made (Figures $4 \mathrm{~A}$ and $5 \mathrm{~A}$ ). The dura was opened in a horseshoe fashion on the left side, using the sinus as the durotomy base and being careful to avoid injuries to the sinus and cortical veins (Figures 4B and 5B). The parietal lobe was then retracted to visualize the cingulate gyrus. Bridging veins were kept intact. Cerebrospinal fluid was drained at this point through the ventriculostomy to further relax the brain, and the cingulate gyrus was gently retracted. This maneuver allowed us to go beneath the gyrus, deep into the callosal sulcus. After confirming the correct location using the neuronavigation probe, we made a small paracallosal incision deep into the callosal sulcus without
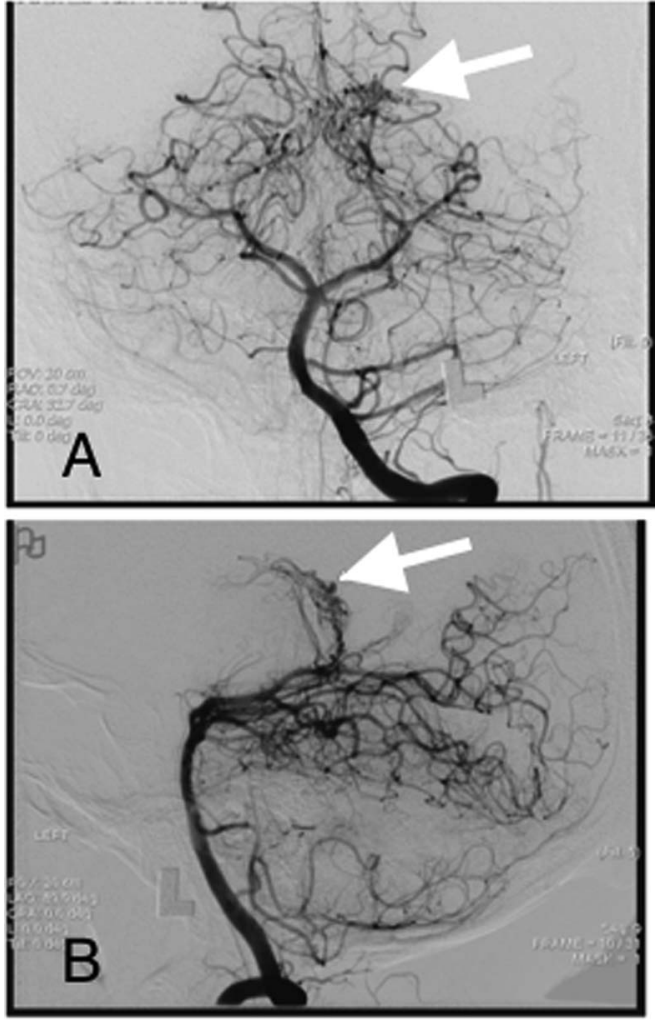

Figure 3. Digital subtraction angiography in (A) anteroposterior and (B) lateral views demonstrates left atrial arteriovenous malformation (arrows).

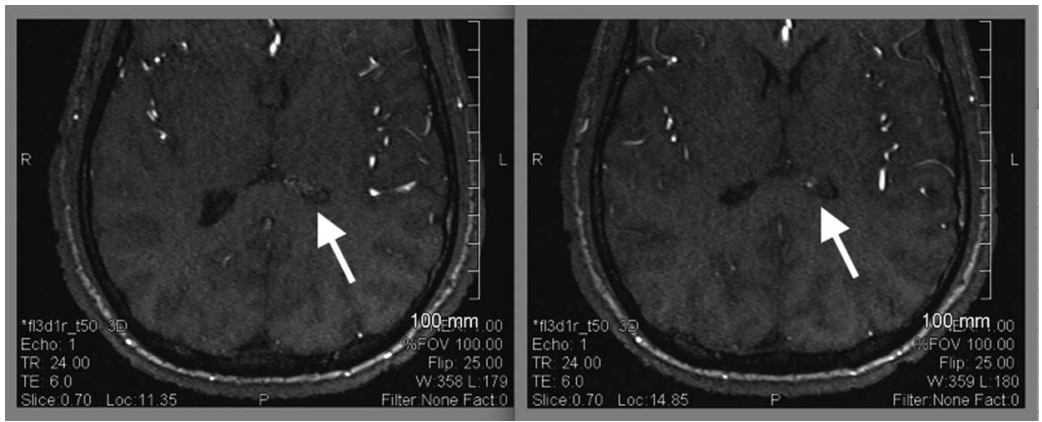

Figure 2. Magnetic resonance angiography axial cuts demonstrate an arteriovenous malformation located in the atrium of the left lateral ventricle (arrows). 

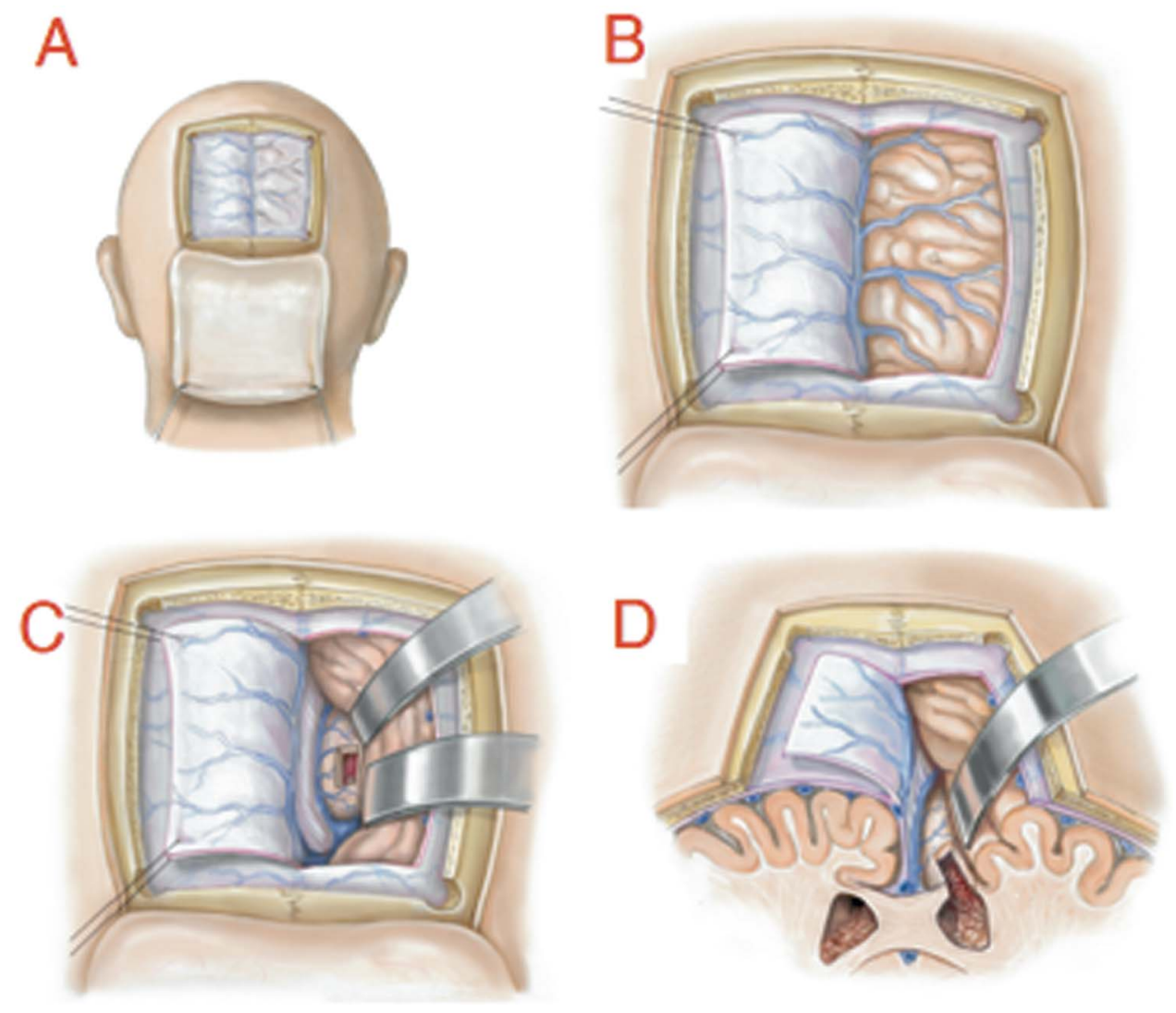

Figure 4. Artist's representation of the surgical approach shows (A) a craniotomy overlying the parietooccipital sagittal sinus, (B) left-sided horseshoe dura incision with the sinus as the durotomy, (C) parietal lobe retraction allowing for adequate visualization of the cingulate gyrus that was gently retracted for deep access to the callosal sulcus, and (D) a small paracallosal incision made deep into the callosal sulcus without transecting the cingulate gyrus.

transecting the cingulate gyrus (Figures 4C, 4D, and 5C). The atrium of the left lateral ventricle was reached without difficulty (Figure 5D). The small AVM was visualized and resected in the usual fashion without intraoperative rupture or hemorrhage. Surgery was completed without complications.

The patient had an uncomplicated postoperative course, and the formal visual field examination remained within normal limits postoperatively. The EVD was removed on postoperative day 2 . On postoperative day 3 , the patient was discharged home without any neurologic deficits. The patient remained neurologically intact at the 2-week and 3-month follow-up examinations.

\section{DISCUSSION}

We present the interhemispheric paracallosal approach for an AVM of the atrium, a new surgical approach to the atrium of the lateral ventricle that avoids the common cingulate gyrus corticotomy and instead uses a small paracallosal (deep into the callosal sulcus) incision to minimize brain tissue disruption.

When operating in or near the lateral ventricles, the surgeon must be cognizant of the optic radiations, including the Meyer loop, that have been found to cover the lateral wall of the temporal horn of the lateral ventricle up to the atrium.
However, the medial wall of the atrium does not consist of any optic radiation fibers. ${ }^{6}$ Mahaney and Abdulrauf ${ }^{6}$ show that the superior parietooccipital approach, the transtemporal approach, and even the relatively new lateral insular transsulcal approach ${ }^{7}$ would all traverse optic radiation fibers. They advocate the posterior interhemispheric parietooccipital approach with a slightly more inferior entry point than the standard protocol to best avoid the optic radiation fibers. ${ }^{6}$ However, their advocated approach (still considered the transcallosal approach) transects fibers of the cingulate gyrus and the splenium of the corpus callosum, so the patient is therefore vulnerable to postoperative complications such as auditory or visual disconnection syndrome.

Our approach to the atrium avoids dividing the cingulate gyrus, thereby preventing potential associated neurologic deficits such as visual field deficits, alexia, and visual anomia. ${ }^{5}$ The cingulate gyrus has been implicated in many complex neurologic tasks, including orienting the body in space, interoception, and visual stream inputs, and has even been proposed to be a "cortical hub," a control center for cognition..$^{8-11}$ In addition, disruption to the cingulate gyrus has been shown to be associated with impaired pain regulation, indifference to pain, apathy, and depression. ${ }^{12}$ 

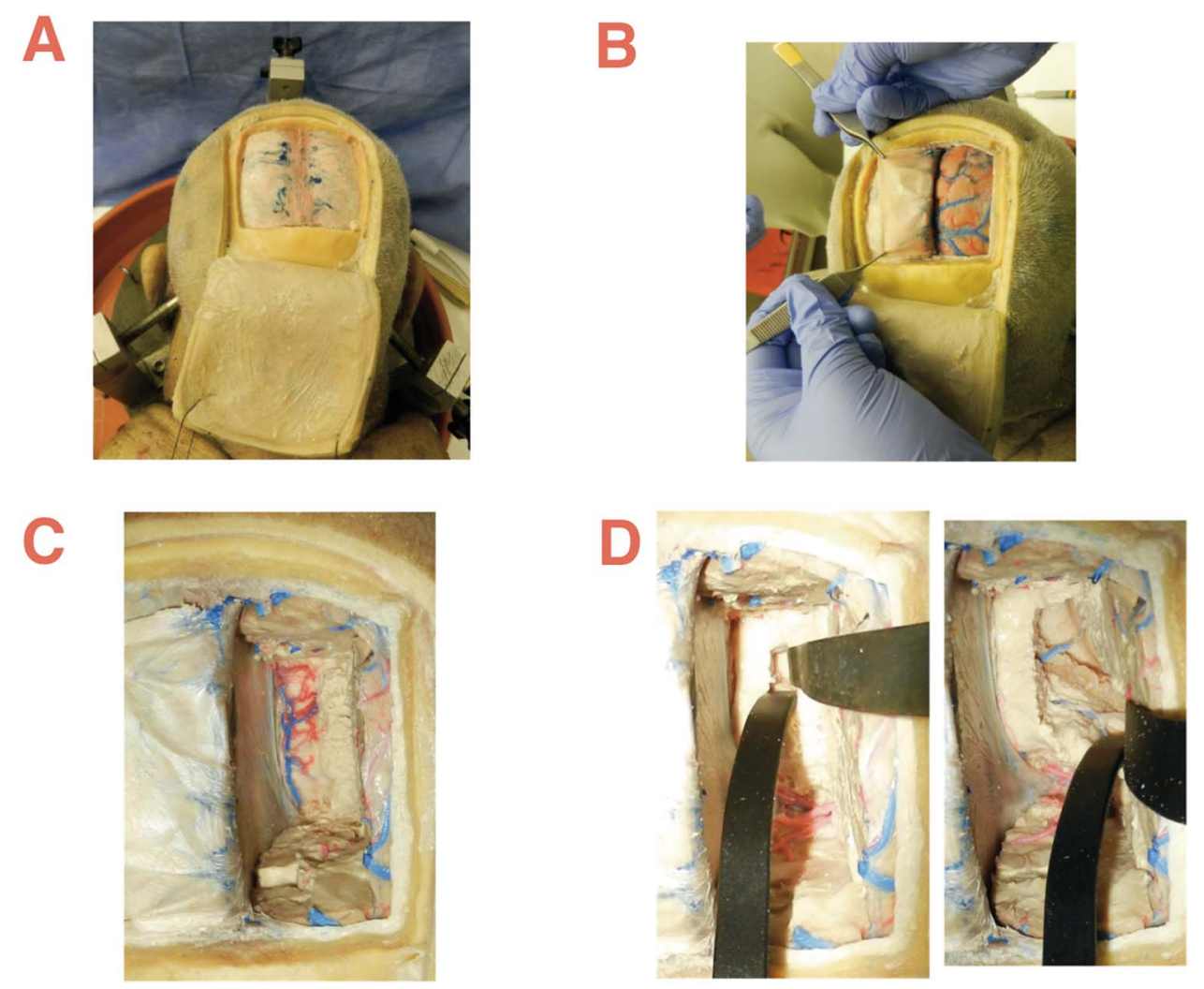

Figure 5. Cadaver dissection corresponding to the illustration in Figure 4 shows (A) parietooccipital craniotomy; (B) dural opening with its base at the superior sagittal sinus, with bridging veins visualized going into the sinus; $(C)$ corpus callosum and vessels that course the cingulate sulcus after removal of the parietooccipital cortex along with the cingulate gyrus; and (D) corticotomy (off midline) made in the depth of the cingulate sulcus to access the ventricular atrium (left panel) and visualization of the atrium with choroid plexus and superior choroidal vein (right panel).

Considering the complex involvement of the cingulate cortex in both neurologic and neuropsychological processes, sparing it, as we have done, when approaching the atrium of the lateral ventricle is advantageous.

In addition, even though the transcallosal approach is well tolerated, ${ }^{13}$ it has neurologic risks including mutism, hemineglect, and contralateral hemiparesis. ${ }^{5}$ The transcallosal approach has also been shown to be associated with auditory or visual disconnection syndrome because of the disruption of splenium fibers. ${ }^{14}$ Because we avoided a posterior callosotomy as previously described in the posterior interhemispheric parietooccipital approach, we did not introduce these kinds of risks to the patient.

In the analysis of new approaches to specific brain areas, the adverse effects as well as benefits must be considered. One potential limitation of this interhemispheric paracallosal approach is that the technique can be used only for relatively small lesions in the atrium. Because the paracallosal incision needs to remain limited, the size of the lesion to be excised is thus restricted. Furthermore, this surgical approach requires adequate brain relaxationgenerally achieved with cerebrospinal fluid drainage and appropriate anesthetic techniques - to retract the cingulate gyrus in a gentle manner and avoid retraction-related injuries. ${ }^{15}$ Finally, this approach is highly dependent on the anatomy of the bridging veins and could carry the risk of venous sinus injury because of the exposure of the venous sinus.

\section{CONCLUSION}

We present a case of a patient with an AVM in the atrium of the lateral ventricle that was resected through a novel microsurgical approach. We used a variation of the conventional interhemispheric parietooccipital approach, making the incision in the paracallosal area, deep into the callosal sulcus. Because we avoided both a cingulate gyrus corticotomy and a posterior callosotomy, we decreased the risk of neurologic deficits associated with those incisions. As such, our patient remained neurologically intact at discharge. More research, including tract dissection and imaging-guided tract analysis, is recommended to evaluate the risks, benefits, and potential unknown complications associated with the described approach.

\section{ACKNOWLEDGMENTS}

The authors have no financial or proprietary interest in the subject matter of this article. The authors would like to acknowledge Ms Barbara Siede for her help in illustration. 


\section{REFERENCES}

1. Ponce FA, Spetzler RF. Arteriovenous malformations: classification to cure. Clin Neurosurg. 2011;58:10-12.

2. Pradilla G, Coon AL, Huang J, Tamargo RJ. Surgical treatment of cranial arteriovenous malformations and dural arteriovenous fistulas. Neurosurg Clin N Am. 2012 Jan;23(1):105-122. doi: 10.1016/j.nec.2011.10.002.

3. Barrow DL, Dawson R. Surgical management of arteriovenous malformations in the region of the ventricular trigone. Neurosurgery. 1994 Dec;35(6):1046-1054.

4. Yasargil MG, Abdulrauf SI. Surgery of intraventricular tumors. Neurosurgery. 2008 Jun;62(6 Suppl 3):1029-1040; discussion 1040-1041. doi: 10.1227/01.neu.0000333768.12951.9a.

5. Omay SB, Joachim B, Piepmeier J. Approaches to lateral and third ventricular tumors. In: Schmidek HH, Roberts DW, eds. Operative Neurosurgical Techniques: Indications, Methods, and Results. Philadelphia, PA: Saunders Elsevier; 2006: 753-771.

6. Mahaney KB, Abdulrauf SI. Anatomic relationship of the optic radiations to the atrium of the lateral ventricle: description of a novel entry point to the trigone. Neurosurgery. 2008 Oct;63(4 Suppl 2):195-202; discussion 202-203. doi: 10.1227/01.NEU.0000313121.58694.4A.

7. Nagata S, Sasaki T. Lateral transsulcal approach to asymptomatic trigonal meningiomas with correlative microsurgical anatomy: technical case report. Neurosurgery. 2005 Apr;56(2 Suppl):E438; discussion E438.
8. de Pasquale F, Sabatini U, Della Penna S, et al. The connectivity of functional cores reveals different degrees of segregation and integration in the brain at rest. Neuroimage. $2013 \mathrm{Apr}$ 1;69:51-61. doi: 10.1016/j.neuroimage.2012.11.051.

9. Leech R, Braga R, Sharp DJ. Echoes of the brain within the posterior cingulated cortex. J Neurosci. 2012 Jan 4;32(1):215-222. doi: 10.1523/JNEUROSCI.3689-11.2012.

10. Vogt BA, Vogt L, Laureys S. Cytology and functionally correlated circuits of human posterior cingulated areas. Neuroimage. 2006 Jan 15;29(2):452-466. doi: 10.1016/j.neuroimage.2005.07.048.

11. Vogt BA, Laureys S. Posterior cingulated, precuneal and retrosplenial cortices: cytology and components of the neural network correlates of consciousness. Prog Brain Res. 2005;150:205-217. doi: 10.1016/S0079-6123(05)50015-3.

12. Hamani C, Mayberg H, Stone S, Laxton A, Haber S, Lozano AM. The subcallosal cingulated gyrus in the context of major depression. Biol Psychiatry. 2011 Feb 15;69(4):301-308. doi: 10.1016/j.biopsych.2010.09.034.

13. Lawton MT, Golfinos JG, Spetzler RF. The contralateral transcallosal approach: experience with 32 patients. Neurosurgery. 1996 Oct;39(4):729-734; discussion 734-735.

14. Jun CL, Nutick SL. Surgical approaches to the intraventricular meningiomas of the trigone. Neurosurgery. 1985 Mar;16(3):416-420.

15. Hans $P$, Bonhomme V. Why we still use intravenous drugs as the basic regimen for neurosurgical anaesthesia. Curr Opin Anaesthesiol. 2006 Oct;19(5):498-503. doi: 10.1097/01.aco.0000245274.69292.ad.

This article meets the Accreditation Council for Graduate Medical Education and the American Board of Medical Specialties Maintenance of Certification competencies for Patient Care and Medical Knowledge. 